Cuadernos de Trabajo Social

ISSN: 0214-0314

\title{
Desarrollo, confiabilidad y validez de una escala multidimensional de informes sociales periciales en Trabajo Social (ISP.TS)
}

\author{
Milton Andrés Contreras Sáez ${ }^{1}$
}

Recibido: 28/09/2020; Revisado: 14/10/2020; Aceptado: 21/04/2021

Resumen. El presente artículo se centra en el desarrollo, confiabilidad y validez de una escala multidimensional de informes sociales periciales en Trabajo Social (ISP.TS). El Informe Social Pericial constituye uno de los instrumentos más utilizados por Trabajadores Sociales en el mundo en diferentes materias legales. Las dimensiones trabajadas fueron el concepto de familia, relaciones familiares, resiliencia familiar, factores protectores y de riesgo, proyecto de vida y entornos familiares. El estudio se efectuó con todos(as) los(as) peritos trabajadores sociales inscritos y activos de la Defensoría regional del Maule, en Chile (15). La confiabilidad se evaluó con análisis de consistencia interna mediante el empleo del coeficiente alfa de Cronbach, así como por el análisis de la homogeneidad de sus ítems examinando la correlación ítem - total corregido. La validez fue analizada mediante el juicio de expertos y a través del coeficiente de correlación de Pearson. La escala obtuvo un coeficiente alfa de Cronbach de 0.904 y la validez del instrumento se realizó por medio de juicio de expertos. La escala ISP.TS es una medida valida y confiable para la evaluación de los informes sociales periciales que puede ser utilizada en diferentes contextos.

Palabras clave: informe social, peritaje social, Trabajo Social, escala multidimensional de informes sociales periciales en Trabajo Social.

Sumario: Introducción. 1. Metodología. 1.1 Participantes. 1.2 Instrumento. 1.3 Procedimiento. 1.4 Análisis de datos. 2. Resultados. 2.1 Análisis de la confiabilidad. 2.2 Validez del instrumento. 3. Conclusiones y discusión. 4. Referencias bibliográficas.

Cómo citar: Contreras Sáez, M. A. (2021) Desarrollo, confiabilidad y validez de una escala multidimensional de informes sociales periciales en Trabajo Social (ISP.TS). Cuadernos de Trabajo Social, 34(2), 353-366.

\section{Introducción}

En el contexto histórico del Trabajo Social, se ha apreciado que el Informe social constituye uno de los instrumentos con mayor ambigüedad dentro de la disciplina. Esto se debe a que el Informe social se ha transformado en uno de los "aprendizajes" más utilizados y solicitados en la investigación para la intervención en cualquier campo de acción donde se desempeñen los trabajadores sociales (Robles, 2013; Quintero, 2014; Méndez, 2015; Honores y Quizhpe, 2019).

Uno de los informes sociales más elaborados y que requieren una alta especialización de los trabajadores sociales es el denominado Informe social pericial (ISP), que se caracteriza por ser un documento escrito que contiene resultados del peritaje social, realizado exclusivamente por un(a) Trabajador(a) Social. En este documento, se exponen técnicamente la situación social de: el peritado, su familia y la relación con su entorno. En términos generales, es un informe técnico, científico y profesional que permitirá tomar decisiones en cuanto a una situación (causa penal o de protección), por lo que se requieren los principios éticos de la profesión. Se ha demostrado que el Informe social pericial debe desarrollarse en concordancia con diferentes técnicas, estrategias e instru-

\footnotetext{
Universidad de Talca; Chile.

E-mail: milton.contreras.saez@gmail.com
} 
mentos, que no siempre son propios del Trabajo Social, sino que, por lo general, lo son de diferentes disciplinas de las Ciencias Sociales, Humanas y de la Salud.

La formación académica y científica de Hispanoamérica en Trabajo Social se ha caracterizado por enseñar generacionalmente el Informe social pericial y cada uno de sus componentes, en los contextos del aula, la experiencia docente, práctica y profesional. Sin embargo, la formación en este tipo de informes no se ha logrado por el aprendizaje basado en la evidencia científica, pese a ser parte de una disciplina que se caracteriza por la actividad científica y que declara la realización de sistematizar experiencias periciales (Hernández, 2017; Quintero, 2017). Ejemplo de ello es que, al intentar realizar una revisión sistemática de literatura científica sobre Informe social pericial con objetivos amplios de conceptualización y metodología, los resultados no son muy alentadores. En una primera fase de identificación de la literatura -siguiendo lineamientos de Sapiro y Ward (2019) - a partir de las palabras clave como "informe social" y "peritaje social" (como tema) en bases de datos especializadas -Web Of Science, Proquest, Scopus, Scielo y Dialnet Plus, sin limitaciones de años- tenemos como resultado solo ocho registros en Informe social (1 en Proquest y 7 en Dialnet Plus), que corresponden a artículos de revisión; y en "peritaje social" son también 8 registros (1 en Proquest, 1 en Scielo y 6 en Dialnet Plus) que corresponden a siete artículos de revisión y uno empírico.

Esta búsqueda corrobora que el abordaje del Informe social pericial, sea cual sea su índole, requiere no solamente un reconocimiento disciplinar, sino también demostrar un producto de carácter científico. Tradicionalmente el Informe social pericial se ha dimensionado como una prueba metodológica de tipo descriptivo de una realidad o situación particular, que se realizaba por medio de la observación/ investigación a través de la entrevista, visita domiciliaria y revisión documental, otorgando como producto final un informe que asegurara un contenido verídico (Antón, 2014; Casas y Niño, 2015; Lijterman, 2015).

El resultado de un Informe social pericial se da gracias a la rigurosidad del o de la profesional a cargo del peritaje, de cómo emplea las diferentes técnicas, estrategias e instrumentos de evaluación y el uso que le da en el texto final como en el juicio oral, según corresponda.
Frente a los escenarios complejos y diversos donde el Trabajo Social se moviliza constantemente, la realización de Informe social pericial que incluya dimensiones adecuadas a la situación del "peritado", permite tomar mejores decisiones tanto a nivel institucional como al del poder judicial (Quintero, 2014).

Pese a que existen algunas recomendaciones internacionales, como por ejemplo del Council on Social Work Education (CSWE), que señala que los y las trabajadores sociales pueden asumir un liderazgo en el uso de la mejor evidencia disponible, por medio de la investigación y evaluación, para diseñar, implementar, monitorear y evaluar intervenciones en temáticas asociadas al Trabajo Social forense que optimicen las prácticas que permitan avanzar hacia un modelo basado en la evidencia, lo que requiere un trabajo mancomunado para lograrlo, que, en términos prácticos, sigue siendo escaso (Rubin, 2008; Maschi, 2017).

Lamentablemente, Trabajo Social carece de instrumentos propios de medición que permitan comprender las multidimensiones que se abordan en el proceso de peritaje social, por lo que, en este estudio se destaca la creación y validación de un cuestionario que aborda dimensiones básicas de comprensión situacional y abordaje en el proceso de redacción de un Informe social pericial, desde una mirada compleja y que responde a los principios básicos del Trabajo Social, como: la búsqueda de la justicia social, el reconocimiento de la diversidad y la creación de propuestas de intervención para un desarrollo social sustentable.

Para ello, es importante realizar sinergia con lo declarado por la Federación Internacional de Trabajo Social:

El Trabajo Social es una profesión basada en la práctica y una disciplina académica que promueve el cambio y el desarrollo social, la cohesión social, y el fortalecimiento y la liberación de las personas. Los principios de la justicia social, los derechos humanos, la responsabilidad colectiva y el respeto a la diversidad son fundamentales para el Trabajo Social. Respaldada por las teorías del Trabajo Social, las Ciencias Sociales, las Humanidades y los conocimientos indígenas, el Trabajo Social involucra a las personas y las estructuras para hacer frente a desafíos de la vida y aumentar su bienestar (Federación Internacional de Trabajadores Sociales, 2014). 
Esta declaración permite realizar un trazado sociohistórico donde paulatinamente los principios orientadores (basados en la praxis) deben prevalecer en cualquier propuesta de investigación e intervención y el Informe social pericial debe demostrarlo. Para lograrlo, algunos autores señalan que es necesario considerar enfoques integradores, especializados y colaborativos en las prácticas que se generan con poblaciones afectadas, en cierta medida, por decisiones políticas y cuestiones legales, con una actuación científica que evite juicios de valor (Maschi y Killian, 2009, 2011; Policarpo, 2017).

La construcción de esta escala resulta clave para Trabajo Social, ya que, en el ámbito de trabajo con familias, siempre se ha propiciado mejorar las relaciones intrafamiliares, las condiciones de vivir en familia y el mejoramiento en las estrategias de intervención, principalmente con grupos focalizados de niños/as, adolescentes y sus familias que están en riesgo social (White, 2007). Por lo tanto, este trabajo basado en la evidencia pretende considerar multidimensiones que permitan comprender las diferentes realidades individuales y familiares y, por consiguiente, es una invitación a que se incorporen en futuras atenciones e intervenciones de manera íntegra y no fragmentada en cualquier materia de familia (Dierckx, Devlieghere y Vandenbroeck, 2020).

La Escala multidimensional de informes sociales periciales en Trabajo Social (ISP.TS) para peritos trabajadores sociales es un instrumento desarrollado como parte de dos proyectos de Innovación y Desarrollo de la docencia de la Universidad Autónoma de Chile, que ha permitido ser una propuesta que enfatiza establecer lineamientos claros y precisos en el análisis y abordaje de dimensiones que interactúan unas con otras en cualquier materia legal. Para poder seleccionar las dimensiones, se elaboró una búsqueda especializada en las bases de datos antes mencionadas, se revisaron los lineamientos técnicos de los sistemas judiciales de Sudamérica y España, como también se entrevistó previamente a académicos e investigadores en la materia. Finalmente, se seleccionaron seis dimensiones:

1. Concepto de familia. Dimensión entendida como captura de la definición de familia de la persona peritada y su familia, y donde se genera la identidad personal y familiar (Cinamon y Rich, 2002; González, 2013).
Por lo tanto, se integra la experiencia de cada peritado, con el uso de conceptualizaciones de familia procedentes de otras disciplinas y ámbitos legales como también del Trabajo Social, y a la vez de la identificación de fortalezas, debilidades y potencialidades que la familia expone dentro de su propio funcionamiento.

2. Las relaciones familiares. Dimensión entendida como poder socializador, proveedor de educación y desarrollo de las personas y como parte de dinámicas complejas (Granic, Hollenstein, Dishion y Patterson, 2003; Muñoz, 2005; Oliva, 2006)during, and after the transition. Boys $(n=149$ families. Se integra con cómo se centra la observación de los roles de los miembros de la familia, y de los vínculos afectivos, el ejercicio de la parentalidad (si corresponde), las relaciones afectivas y sociales, nuevas dinámicas familiares, los patrones de interacciones, valores, las creencias, costumbres, la comunicación, el apoyo y afecto que se dan dentro del sistema familiar.

3. La resiliencia familiar. Dimensión comprendida como un conjunto de procesos que activa a una familia en un momento de estrés ( Villalba, 2003; Gómez y Kotliarenco, 2010). En este estudio centrado en verificar si se observan las potencialidades, capacidades de superación, la búsqueda de la autorregulación personal y familiar como también el cumplimiento de metas de los peritados.

4. Los factores protectores y de riesgo. Dimensión entendida como situaciones que favorecen o desfavorecen el desarrollo óptimo de una persona y su familia, como también las relaciones en su contexto ( Montañés y Bartolomé, 2007, Bartolomé, Montañes, Rechea y Montañez, 2010). Se centra en la consideración de vínculos relevantes al interior de la familia, estilos de comunicación, estilos de resolución de conflictos, y vínculos con redes externas, identificación de factores de riesgo, tanto históricos (estáticos) como actuales (dinámicos).

5. El proyecto de vida (individual y familiar). Dimensión entendida como el anhelo de una persona (o familia) en un momento determinado y permeado por vivencias personales, familiares y contextuales ( Medán, 2013; Contreras, 2018, 2020; Santana-Vega, Medina-Sánchez y Feliciano-García, 
2019). En este caso se centra en considerar las posibilidades, factibilidades y oportunidades de su contexto sociocultural de contar con un proyecto de vida, como también las posibilidades de autoexpresión y autodesarrollo. Por la población donde se realizan las pericias, se integra una aseveración que indica si la pena sustitutiva posibilita su proyecto de vida.

6. Los entornos familiares. Dimensión explicitada como espacios micro y exosistémicos donde se encuentra y moviliza una persona y su familia, que puede afectar o complicar el desarrollo biopsicosocial (Oliva, Parra, Sánchez-Queija y López, 2007; Calvete, Orue y Sampedro, 2011). En este estudio se consideraron los riesgos del entorno y vecindario, estabilidad de la vivienda, relaciones sociales y conexión cívica de los peritados.

En Latinoamérica, no se ha creado ni validado ningún instrumento para evaluar los informes sociales periciales. Con el afán de contribuir a la disciplina, específicamente para los profesionales de la intervención como en proceso formativo en ámbitos familiares, se realizó el presente estudio. El objetivo fue evaluar la confiabilidad de los ítems que integran el instrumento de medición y que reflejen una fuerte correlación entre ellos (DeVellis, 1991) y la validez de medir efectivamente cada variable que integra un Informe social pericial, lo cual se realizó por medio de la Escala multidimensional de informes sociales periciales en Trabajo Social (ISP.TS). Se optó por una escala de auto-reporte con modalidad de respuestas de tipo Likert con las dimensiones antes mencionadas. Estas son las necesarias para trabajar en un proceso de investigación e intervención, sea cual sea su materia, y que, por lo demás, se cuenta con amplia literatura científica que la avala; y se utilizó el juicio de expertos que permitió la validez de contenido. Por otro lado, se invita a que en investigaciones futuras se podría emplear esta escala para favorecer la comprensión de los informes sociales periciales en cualquier parte del mundo.

\section{Metodología}

\subsection{Participantes}

Las personas participantes en el presente estudio son peritos trabajadores sociales. Para la definición de la muestra de peritos se tomó contacto con todos los inscritos y activos en la Defensoría Penal Pública de la Región del Maule. La inclusión solo de estos peritos se debe a que esta es la institución pública que presta servicios en diferentes materias judiciales, y para seleccionar a profesionales se rigen por la revisión de altos estándares de calidad en formación académica, especialización en temáticas asociadas y años de experiencia.

En el proceso de pilotaje participaron 9 peritos trabajadores sociales que ejercen su labor en el ejercicio libre de la profesión y que, como criterio de inclusión, debían llevar por lo menos 5 años realizando peritajes sociales. Luego del análisis de la información, cuatro académicos expertos en la materia de Chile y Argentina colaboraron en realizar ajustes en el lenguaje, como también la eliminación de dos aseveraciones en la dimensión de resiliencia y dos del entorno familiar.

Luego de las modificaciones, se tomó contacto con los sujetos incluidos en el estudio final. Estos fueron los 15 peritos trabajadores sociales, inscritos y activos de la Defensoría Penal Pública de la Región del Maule (todos y todas participaron). El $80 \%$ de peritos son de género femenino, mientras el $20 \%$ restante son masculino. Respecto al tiempo como peritos, el 7\% lleva entre 6 meses y 1 año, el 13\% entre 1 y 2 años, el $7 \%$ entre 3 y 4 años, el 13\% entre 4 y 5 años y el $60 \%$ más de 5 años. La aplicación de la escala fue realizada por correo electrónico y completada por cada perito.

\subsection{Instrumento}

La escala multidimensional de informes sociales periciales en Trabajo Social (ISP.TS) (Anexo 1) es un instrumento diseñado para evaluar el uso de las dimensiones utilizadas en el Peritaje social, que fue creada en el contexto de dos fondos de innovación y desarrollo de la docencia entre los años 2018 y 2020. Es de auto-reporte con formato respuesta tipo Likert, donde cada ítem consta de cuatro opciones de respuestas (nunca, a veces, casi siempre y siempre). Consta de 32 ítems, que se agrupan en 6 subescalas:

A. Concepto de familia (6): experiencias histórico-sociales, conceptualización de otras disciplinas, concepción de las familias, conceptualización de Trabajo Social, identificación de fortalezas, y conceptualización legal. 
B. Relaciones familiares (6): roles de la familia, parentalidad, relaciones afectivas y sociales, dinámicas y conflictos, patrones de interacción, y comunicación.

C. Resiliencia familiar (4): potencialidades, proceso de superación, fuentes de fortalezas, y reconocimiento del potencial.

D. Factores protectores y de riesgo (6): vínculos y comunicación, factores de riesgo estáticos, redes de apoyo, habilidades personales y sociales, factores de riesgo dinámicos, y capacidades.

E. Proyecto de vida (6): factibilidades y oportunidades, posibilidades, restablecimiento de redes, historia de vida, contexto sociocultural, y áreas de autoexpresión y autodesarrollo.

F. Entornos familiares (4): vecindario, estabilidad de la vivienda, interacción con otros, y conexión con el entorno.

Con objeto de asegurar tanto la validez de contenido como la aplicabilidad del instrumento en esta primera versión, inicialmente se estableció una revisión de literatura en un plazo de cuatro meses, donde se logró establecer el contenido más relevante para el estudio del Informe social pericial (1); luego se seleccionaron las variables que mejor se identifican y son necesarias en la pericia, en tres meses (2); para que finalmente fuese sometida a un proceso de depuración por medio de juicio de expertos, en dos meses (3). El criterio para elegir a los expertos fue: ser expertos académicos y prácticos en materia de Informe social pericial, contar con un nivel de postgrados o fueran investigadores activos en la materia, ser de habla hispana y tener disponibilidad para reuniones telemáticas o presenciales. Finalmente, participaron 4 académicos especialistas en Peritaje social, tres de Chile y uno de Argentina, lo que permitió complejizar las perspectivas teóricas, seleccionar las variables y analizar la adecuación de los ítems a la dimensión que evaluaban mediante una escala Likert de cuatro puntos. Se utilizó el acuerdo inter-jueces para eliminar los ítems más problemáticos (Escobar-Pérez y Cuervo-Martínez, 2008; Cabero e Infante, 2014). A nivel estadístico, se obtuvo un coeficiente alfa de Cronbach de 0.904, lo que indica que es excelente (George y Mallery, 2019, p. 231).

\subsection{Procedimiento}

La información fue recogida con un cuestionario anónimo, autoaplicado, previamente ensayado en una prueba piloto. La participación de los peritos trabajadores sociales fue autorizada por la Defensoría Penal Pública del Maule, quienes aceptaron voluntariamente responder el cuestionario. La aplicación del cuestionario fue por vía online por Google Forms (plataforma que cuenta con política de privacidad segura $^{2}$ ), luego de que cada perito firmara un consentimiento informado. En el momento que aceptaron participar, los peritos completaron un apartado de información sociodemográfica (género, comuna y tiempo como peritos) y luego las dimensiones del instrumento antes mencionadas.

\subsection{Análisis de datos}

Los análisis estadísticos se realizaron mediante el programa SPSS 25.0. La evaluación de la confiabilidad del ISP.TS se llevó a cabo en base al análisis de la consistencia interna, mediante el empleo del Coeficiente alfa de Cronbach, así como por el análisis de la homogeneidad de sus ítems, examinando la correlación ítem -total corregido-. La validez de contenido fue analizada mediante el juicio de expertos y la confiabilidad, a través del coeficiente de correlación de Pearson.

\section{Resultados}

La primera parte consistió en revisar que las y los peritos trabajadores sociales hubieran respondido el cuestionario en su totalidad. Posteriormente se realizó un análisis univariado de los datos estadísticos descriptivos de los ítems, agrupados en sus dimensiones. Posteriormente se analizaron las correlaciones entre los ítems de la escala inicial por conceptos y se mostró que un total de treinta y dos ítems no presentaban correlaciones significativas (véase el anexо 2).

La política de privacidad de Google indica que incluyen funciones de seguridad sólidas que protegen constantemente la información y que además puede detectar y bloquear automáticamente amenazas de seguridad para que nunca afecten. Si se detecta algún riesgo; se podrán en contacto e indicarán los pasos a seguir para mejorar la protección. Enlace: https://policies.google.com/ privacy\#infosecurity 
Específicamente, en el concepto de familia existen 6 ítems que resaltan por sus bajas correlaciones. En los ítems experiencias histórico-sociales con conceptualización de Trabajo Social se muestra una relación positiva más significativa que el resto $(0,60)$ de las correlaciones. En cuanto a la relación con los otros ítems también muestran resultados no significativos, excepto por los ítems de conceptualización legal. En las relaciones familiares, existen 6 ítems que tienen correlaciones no significativas con el resto de las dimensiones, excepto el ítem parentalidad con dinámicas y conflictos, donde mantiene una relación directa del 0,92. En los factores protectores y de riesgo, y proyecto de vida tienen 6 ítems cada uno, mientras que resiliencia familiar y por último entorno familiar tienen 4 ítems respectivamente. Si bien existen ítems con correlaciones negativas, estas no son significativas, pero sí hay correlaciones que son positivas entre ítems, como por ejemplo relaciones familiares (parentalidad) con factores protectores y de riesgo (riesgo dinámico), concepto de familia (legal) con entornos familiares (interacciones con otros y conexión con el entorno).

\subsection{Análisis de la confiabilidad}

El análisis de fiabilidad de cada una de las dimensiones y del cuestionario global se rea- lizó por medio del análisis de la consistencia interna del ISP.TS, específicamente mediante el coeficiente alfa de Cronbach, cuyo valor fue de 0,904; lo cual resulta excelente. Como criterio general, George y Mallery sugieren lo siguiente para evaluar los coeficientes de alfa de Cronbach: Coeficiente alfa $>.9$ es excelente; Coeficiente alfa $>.8$ es bueno; Coeficiente alfa $>.7$ es aceptable; Coeficiente alfa $>.6$ es cuestionable; Coeficiente alfa $>.5$ es pobre; y Coeficiente alfa $<.5$ es inaceptable (2019)

El análisis de la homogeneidad de los ítems, efectuado a través de las correlaciones ítem-total corregido, puede observarse en la Tabla 1. Más del 90\% de los ítems tuvo correlación mayor o igual a $0,25-0,30$, lo cual indica que tienen buena capacidad discriminativa y contribuyen significativamente con la puntuación total del ISP.TS. Como se aprecia en la misma tabla, no es necesario eliminar los ítems de concepto familia Agentes y fortalezas, resiliencia recopilación de información, factores protectores riesgos protectores y proyecto de vida, que presentan menor capacidad discriminativa, ya que su eliminación no aumentaría en forma importante la confiabilidad (Alfa de Cronbach) del instrumento en su totalidad, lo que refuerza el trabajo realizado previamente por medio del juicio de expertos en la validez de contenido.

Tabla 1. Estadísticas de total de elementos del ISP.TS

\begin{tabular}{|c|c|c|c|c|c|}
\hline & Ítems ISP.TS & M.E.S. & V.E.S. & C.E.C. & A.C.S. \\
\hline 1 & $\begin{array}{l}\text { Tomo en cuenta las experiencias histórico-sociales de los } \\
\text { agentes en cuestión, para entender como construyen su pro- } \\
\text { pia conceptualización de familia. }\end{array}$ & 113,27 & 114,781 & ,319 & ,903 \\
\hline 2 & $\begin{array}{l}\text { Utilizo conceptualizaciones de familia desde otras discipli- } \\
\text { nas para articular con las que tienen las propias familias en- } \\
\text { trevistas. }\end{array}$ & 113,60 & 111,971 & ,334 & ,904 \\
\hline 3 & $\begin{array}{l}\text { Incorporo la concepción de familia de los agentes en cues- } \\
\text { tión para la construcción de la Pericia Social. }\end{array}$ & 113,40 & 115,686 & ,129 & ,906 \\
\hline 4 & $\begin{array}{l}\text { Utilizo conceptualizaciones de familia desde el Trabajo So- } \\
\text { cial para articular con las que tienen las propias familias en- } \\
\text { trevistas. }\end{array}$ & 113,53 & 112,552 & ,297 & ,904 \\
\hline 5 & $\begin{array}{l}\text { Identifico las fortalezas, potencialidades y debilidades que la } \\
\text { familia expone dentro de su propio funcionamiento. }\end{array}$ & 113,13 & 119,124 &,- 250 & ,907 \\
\hline 6 & $\begin{array}{l}\text { Utilizo conceptualizaciones de familia desde el ámbito legal } \\
\text { articulando con las de los propios agentes en cuestión. }\end{array}$ & 113,60 & 101,829 &, 818 & ,894 \\
\hline 7 & $\begin{array}{l}\text { Me centro en los roles de los miembros de la familia, ge- } \\
\text { nerando énfasis en los vínculos afectivos que las personas } \\
\text { mantienen en su contexto familiar. }\end{array}$ & 113,13 & 116,124 & ,286 & ,904 \\
\hline
\end{tabular}


8 Observo los ejercicios de parentalidad, toma de decisiones, y vínculos que se producen en la familia en los casos que corresponda.

9 Observo las relaciones afectivas y sociales que se mantienen dentro del sistema familiar de cohabitación

10 Analizo como emergen nuevas dinámicas familiares y áreas de conflictos potenciales, estudiando las relaciones entre sus miembros en vinculación con su medio externo

11 Focalizo en la relevancia de los patrones de interacción que los miembros de la familia establecen, observando los sistemas de valores, creencias y costumbres.

12 Valoro la comunicación, el apoyo y el afecto mutuo, además de las situaciones de hostilidad o conflicto dentro del sistema familiar del agente en cuestión

13 Me centro en recopilar información y transformarla en fundamentos que potencien las capacidades de los agentes en cuestión, aspecto que destaco en la apreciación profesional.

14 Visualizo la resiliencia de los agentes en cuestión como un proceso de superación de la adversidad y búsqueda de la autorrealización personal.

15 Oriento mi observación en las fuentes de fortaleza, apoyo y resiliencia familiar de los agentes en cuestión.

16 Organizo la información del agente en cuestión desde el reconocimiento del potencial de cada persona para alcanzar sus metas

17 Considero relevantes los vínculos al interior de la familia, estilos de comunicación, estilos de resolución de conflictos y vínculos con redes externas.

18 Realizo proposiciones atendiendo a los factores de riesgos estáticos identificados.

19 Me centro en descubrir redes de apoyo que permitan intervenir en los factores de riesgo del infractor de ley.

20 En la recopilación de antecedentes individuales, familiares y penales, relevando el fortalecimiento de habilidades personales y sociales del infractor de ley.

21 Realizo proposiciones de acuerdo con los factores de riesgos dinámicos identificados.

22 Visualizo la importancia de reconocer los factores protectores con la finalidad de que estos potencien las capacidades del agente en cuestión

23 Considero las posibilidades, factibilidades y oportunidades para la construcción de proyecto de vida del agente en cuestión.

24 Considero que, a través de la obtención de una pena sustitutiva, se posibilita desarrollar el proyecto de vida de una persona infractora de ley

25 Considero que como perito Trabajador Social puedo contribuir al restablecimiento de las redes necesarias para los

113,33

$111,524 \quad, 471$

,901

113,33

$109,810 \quad, 612$

,899

113,73

$103,781 \quad, 801$

, 895

113,47

$110,552,654$

,899

113,20

$114,314,444$

,902

113,13

117,838

$-, 022$

,906

$113,13 \quad 114,838 \quad, 520$

,903

113,27

$110,495 \quad, 817$

,898

113,33

$111,524 \quad, 471$

,901

113,13

$114,838 \quad, 520$

,903

113,67

106,667 ,459

,903

113,53

$108,552 \quad, 400$

,904

$113,20 \quad 113,457 \quad 560 \quad 901$

$113,67 \quad 103,667 \quad 655$

,897

113,33

$115,524,149$

,906

113,40

$108,400 \quad, 700$

, 898

113,67

$102,810 \quad, 766$

,895

114,07

106,210 ,440

,904 proyectos de vida de las personas condenadas a Penas Sustitutivas 
26 Considero la historia de vida y expresión de lo que desea ser a futuro el agente en cuestión al momento de construir la Pericia Social

27 En las Pericias Sociales valoro los proyectos de vida de los agentes en cuestión en base a su propio contexto sociocultural

28 Vislumbro las áreas de autoexpresión y autodesarrollo, relaciones interpersonales, relaciones sociales y vida profesional del agente en cuestión.

29 Considero los riesgos del entorno y del vecindario como circunstancias de vida donde se encuentra inmersa la familia del agente en cuestión.

30 Reflexiono sobre la importancia de la estabilidad de la vivienda del agente en cuestión como factor protector

31 Observo las relaciones sociales de la familia del agente en cuestión en su interacción con otras personas y en diferentes $113,13 \quad 114,838 \quad, 520$ ,903 espacios del mismo entorno

32 Valoro que la conexión con su entorno permite mayor incidencia en asuntos cívicos, culturales y de toma de decisiones

$\begin{array}{llll}113,20 & 116,886 & , 101 & , 905 \\ 113,40 & 112,543 & , 371 & 903\end{array}$

$113,47 \quad 108,124 \quad 704 \quad 898$

$113,67 \quad 109,095 \quad 375 \quad, 904$

$113,47 \quad 103,552 \quad, 723 \quad, 896$

$113,47 \quad 106,124 \quad 643$

, 898

M.E.S: Media de escala si el elemento se ha suprimido. V.E.S: Varianza de escala si el elemento se ha suprimido. C.E.C: Correlación total de elementos corregida. A.C.S: Alfa de Cronbach si el elemento se ha suprimido.

\subsection{Validez del instrumento}

La validez del instrumento basado en el juicio de expertos se realizó gracias a que reunía las características de no tener información disponible de escalas que permitan evaluar los informes sociales periciales. Además, los expertos eran representantes de Chile y Argentina, estando físicamente dispersos. La visión de expertos sobre el Informe social pericial se realizó a partir de preguntas y de revisión de avances del instrumento, siendo un método donde se logró obtener y depurar la información, acordando los elementos que contienen la Escala. Por otro lado, la ventaja fue que se tuvieron retroalimentaciones y procedimientos periódicos (Cabero e Infante, 2014). El trabajo sistemático previo a la creación del instrumento, su desarrollo y puesta en marcha, permitió que como resultado la ISP.TS tiene una importante confiabilidad (Alfa de Cronbach) y un alentador campo de estudio futuro.

\section{Conclusiones y discusión}

La escala multidimensional de informes sociales periciales en Trabajo Social (ISP.TS) es un instrumento que ha demostrado estar bien diseñado, poseer adecuada confiabilidad y va- lidez. Esta investigación confirma que para los trabajadores sociales que realizan peritajes sociales, el instrumento tiene excelentes propiedades psicométricas, presentando una consistencia interna satisfactoria y una excelente homogeneidad de sus ítems.

En relación a su confiabilidad (coeficiente alfa de Cronbach de 0,904), pese a ser un excelente resultado, no pueden ser comparables aun con otros estudios, debido a que es la primera escala que mide el contenido de los peritajes sociales en el mundo. Para esto, los trabajadores sociales que realicen informes sociales periciales podrán utilizar una o varias dimensiones del instrumento como uso práctico y según las diferentes materias en que se desee aplicar, debido al amplio espectro donde se utiliza el Informe social pericial en contextos sociojurídicos. No obstante, en contexto de investigación, se recomienda utilizar todas las dimensiones observadas, ya que el alfa de Cronbach es excelente en su totalidad y no de manera separada.

Este estudio tiene como limitación, el haber sido llevado a cabo en una población determinada del país (Región del Maule) y con altas exigencias técnicas (Peritos inscritos y activos de la Defensoría Penal Pública). A pesar del tamaño reducido de la muestra (15), se debe considerar, en próximos estudios, que cuando 
se utilice la Escala, ya sea con sus ajustes lingüísticos como contextuales y se aumente el tamaño muestral, se pueda trabajar también en diferentes regiones de un país o incorporar a peritos que realicen funciones en el ejercicio libre de la profesión. Otra limitación es la utilización de información obtenida a través de una escala de auto-reporte, que pese a contar con el juicio de expertos en su revisión y ajuste, como también la contrastación con prueba piloto, puede generar algunos cuestionamientos. Sin embargo, esto no invalida su empleo, ya que las propiedades psicométricas señaladas fueron excelentes, por lo que también se podría aplicar a muestras mayores de peritos trabajadores sociales, donde el instrumento también permitirá medir dimensiones pertinentes que debe contener un Informe social pericial en cualquier materia judicial. Otra limitación es que, al ser la primera escala, se debe optar porque en el futuro se pueda mejorar el análisis psicométrico avanzado como, por ejemplo, comprobar la estructura de la escala con análisis factoriales exploratorias y confirmatorias.

Pese a que la escala ISP.TS es novedosa, dada la experiencia histórica de trabajadores sociales en ámbitos socio-jurídicos, aunque sin un instrumento que permita comprender cuáles son las principales dimensiones que se deben trabajar en el contexto del peritaje, la escala aún no permite describir un puntaje de corte, por lo que sería interesante complejizarla y que, en futuras investigaciones, se pueda determinar un puntaje de corte que permita distinguir explícitamente cuáles son las dimensiones que requieren mayor especialización los(as) peritos(as) trabajadores(as) sociales, como también reforzar las que se incorporan en la pericia social. Además, permitiría distinguir cuáles son los que se deben emplear más en diferentes contextos como, por ejemplo, en protección de niños y adolescentes, restitución de derechos, causas de sanción, revocación o penas sustitutivas de personas imputadas. Por otro lado, es importante destacar a la población participante, que el uso de la escala no es para evaluar si están realizando bien o mal su trabajo, sino el contenido del Informe social pericial.

El disponer de este instrumento es un aporte para investigar en profundidad el Peritaje social y, en especial el Informe social pericial, como un insumo disciplinar histórico que requiere una mayor complejidad basada en la evidencia, que además servirá para comprobar aspectos métricos de esta escala y de su aplicación práctica. Por otro lado, podría apoyar al diseño de una enseñanza más especializada y acorde con los escenarios emergentes de los y las estudiantes de Trabajo Social en cualquier parte del mundo, como también de las y los trabajadores(as) que ejercen la profesión. Por lo tanto, poder profundizar en búsquedas bibliográficas de investigación similares e invitar a profesionales e investigadores a indagar en este constructo; y pese a que este trabajo se hace por medio de una escala, no sea necesariamente con el enfoque cuantitativo, sino también con el enfoque cualitativo, ya sea de manera integrada o separada.

Finalmente, los resultados obtenidos en este estudio confirman que la Escala multidimensional de informes sociales periciales en Trabajo Social (ISP.TS) es un instrumento válido y confiable para la evaluación de los informes sociales periciales que incluye las dimensiones que interactúan en cualquier materia legal (concepto de familia, relaciones familiares, resiliencia familiar, factores protectores y de riesgo, proyecto de vida y entornos familiares), teniendo presente que su confiabilidad fue excelente (coeficiente alfa de Cronbach de 0,904). De igual modo, se recomienda que, en futuras investigaciones, se puedan mejorar los métodos del procedimiento utilizado que permitan reducir subjetividades y que, efectivamente, se comprenda que su contenido se asocia a la evaluación de los informes sociales periciales y no a la labor de los peritos; así como también para realizar estudios comparativos por contextos sociojurídicos y también socioculturales.

\section{Referencias bibliográficas}

Antón, Y. (2014). Trabajo Social en los juzgados de familia [Social Work in the family courts]. Trabajo Social Hoy, 71 (Primer Cuatrimestre), 97-114. Doi: https://doi.org/10.12960/tsh.2014.0006

Bartolomé, R., Montañes, M., Rechea, C. y Montañez, J. (2010). Los Factores de Protección frente a la Conducta Antisocial:¿Explican las diferencias en violencia entre chicas y chicos? Revista Española de 
Investigación Criminológica (REIC), 7(8), 1-15. Recuperado de: http://www.criminologia.net/pdf/reic/ ano7-2009/a72009art3.pdf

Cabero, J. e Infante, A. (2014). Empleo del método Delphi y su empleo en la investigación en comunicación y educación. Edutec. Revista Electrónica de Tecnología Educativa, 48, 1-16. Recuperado de: http:// www.edutec.es/revista/index.php/edutec-e/article /view/187

Calvete, E., Orue, I. y Sampedro, R. (2011). Violencia filio-parental en la adolescencia: Características ambientales y personales. Infancia y Aprendizaje, 34(3), 349-363. Doi: https://doi. org/10.1174/021037011797238577

Casas, A. y Niño, M. (2015). Incorporación del peritaje social desde el análisis de género a los procedimientos en materia familiar, interacciones de un trabajo social contemporáneo. Revista Trabajo Social, $0(9), 59-71$.

Cinamon, R.G. y Rich, Y. (2002). Profiles of attribution of importance to life roles and their implications for the work-family conflict. Journal of Counseling Psychology, 49(2), 212-220. Doi: https://doi. org/10.1037/0022-0167.49.2.212

Contreras, M. (2018). Dimensiones del Proyecto de vida en Intervenciones Familiares. Investigación/Intervención desde perspectiva adolescente en Centros Residenciales de la Región del Bio Bio. Revista Electrónica de Trabajo Social (18), 94-103. Recuperado de: http://www.revistatsudec.cl/wp-content/ uploads/2015/08/9-Dimensiones-del-Proyecto-de-vida-en-Intervenciones-Familiares.pdf

Contreras, M. (2020). Proyecto de vida: Propuesta de Investigación/Intervención Psicoeducativa no normativa en adolescentes. En: P. Concha y F. García (Eds.), La adolescencia hoy: Problemas y soluciones para terapeutas (pp. 57-75). La Serena, Chile: Nueva Mirada Ediciones.

DeVellis, R. (1991). Scale development: Theory and applications. California. Sage Publications.

Dierckx, M., Devlieghere, J. y Vandenbroeck, M. (2020). Proportionate universalism in child and family social work. Child and Family Social Work, 25(2), 337-344. Doi: https://doi.org/10.1111/cfs.12689

Escobar-Pérez, J. y Cuervo-Martínez, Á. (enero, 2008). Validez De Contenido Y Juicio De Expertos: Una Aproximación a Su Utilización. Avances En Medición, (6), 27-36.

Federación Internacional de Trabajadores Sociales. (Julio, 2014). Definición Global de Trabajo Social. Federación Internacional de Trabajadores Sociales. Recuperado de: https://www.ifsw.org/what-is-socialwork/global-definition-of-social-work

George, D. y Mallery, P. (2019). IBM SPSS Statistics 26 step by step: A simple guide and reference. Londres: Routledge.

Gómez, E. y Kotliarenco, M.A. (2010). Resiliencia Familiar: Un Enfoque de Investigación e Intervención con Familias Multiproblemáticas. Revista de Psicología [revista en Internet]. Revista de Psicología, 19(2), 103-131. Doi: https://doi.org/10.5354/0719-0581.2010.17112 [Consultada el 02 de diciembre de 2019].

Gonzalez, H. (2013). La producción científica sobre la familia en Chile: miradas desde la antropología feminista. La Ventana, 4(38), 88-119. Recuperado de: http://www.scielo.org.mx/scielo.php?script=sci arttext\&pid=S1405-94362013000200005\%0Ahttps://revistas.ucm.es/index.php/RASO/article/download $/ 52629 / 48375 \% 0 \mathrm{~A} \% 0 \mathrm{~A}$

Granic, I., Hollenstein, T., Dishion, T. J. y Patterson, G. R. (2003). Longitudinal Analysis of Flexibility and Reorganization in Early Adolescence: A Dynamic Systems Study of Family Interactions. Developmental Psychology, 39(3), 606-617. Doi: https://doi.org/10.1037/0012-1649.39.3.606

Hernández, A. (2017). Los Trabajadores Sociales como Peritos-Forenses. Actuación desde el ejercicio liberal de la profesión. En: S. Amaro y C. Krmpotic, Diccionario Internacional de Trabajo Social en el Ámbito Socio-Jurídico (pp. 613-618). Barcelona: Nova Casa Editorial.

Honores, B.A. y Quizhpe, J.M. (2019). El peritaje desde la perspectiva del trabajo social. Revista Conrado, 15(68), 267-274. Recuperado de: http://conrado.ucf.edu.cu/index.php/conrado

Lijterman, E. (2015). ¿Quiénes escriben los informes sociales? Un análisis de la conflictividad y el malestar en el despliegue de la 'técnica': sistematización de una experiencia de intervención. Documentos de Trabajo Social, (56), 241-261.

Maschi, T. (2017). Trabajo Social Forense: Conceptos fundamentales. En: S. Amaro y C. Krmpotic, Diccionario Internacional de Trabajo Social en el Ámbito Socio-Jurídico (pp. 223-262). Barcelona: Nova Casa Editorial.

Medan, M. (2012). “Proyecto de vida”? tensiones en un programa de prevención del delito juvenil. Revista Latinoamericana de Ciencias Sociales, Niñez y Juventud, (10), 79-91. 
Méndez, M. (2015). Trabajo Social con Personas y Familias: Modelo Clínico de Intervención. Buenos Aires, Argentina: Editorial Espacio.

Montañés, M. y Bartolomé, R. (2007). Conducta antisocial en adolescentes: Diferencias entre chicos y chicas. Ensayos: Revista de la Facultad de Educación de Albacete, (22), 279-294.

Muñoz, A. (2005). La Familia como contexto de desarrollo infantil: dimensiones de análisis relevantes para la intervención educativa y social. Portularia: Revista de Trabajo Social, 5, 147-163.

Oliva, A. (2006). Relaciones familiares y desarrollo adolescente. Anuario de Psicologia, 37(3), 209-223.

Oliva, A., Parra, Á., Sánchez-Queija, I. y López, F. (2007). Estilos educativos materno y paterno: evaluación y relación con el ajuste adolescente. An. Psicol, (23), 49-56.

Policarto, A. (2017). La familia en la contemporaneidad: Reflexiones para la actuación profesional en el campo sociojurídico. En: S. Amaro y C. Krmpotic, Diccionario Internacional de Trabajo Social en el Ámbito Socio-Jurídico (pp. 167-186). Barcelona: Nova Casa Editorial.

Quintero, A. (2017). El Trabajo Social Forense en el hemisferio iberoamericano: tradiciones, matices y constructos epistemológicos. En: S. Amaro y C. Krmpotic, Diccionario Internacional de Trabajo Social en el Ámbito Socio-Jurídico (pp. 245-262). Barcelona: Nova Casa Editorial.

Quintero, A. (2014). Peritaje social: Contexto no clínico en la intervención socio-familiar. Revista de la Facultad de Trabajo Social, 30(30), 25-40.

Rubin, A. (2008). Practitioners guide to using research for evidence-based practice. Nueva York: Wiley Publishers.

Robles, C. (2013). El informe social forense. Aciertos y debilidades de la intervención profesional. Trabajo Social en el Campo Jurídico. Buenos Aires, Argentina: Editorial Espacio.

Santana-Vega, L.E., Medina-Sánchez, P.C. y Feliciano-García, L. (2019). Proyecto de vida y toma de decisiones del alumnado de Formación Profesional. Revista Complutense de Educación, 30(2), 423-440. Doi: https://doi.org/10.5209/rced.57589

Sapiro, B. y Ward, A. (2019). Marginalized Youth, Mental Health, and Connection with Others: A Review of the Literature. Child and Adolescent Social Work Journal, 1-15. Doi: https://doi.org/10.1007/s10560019-00628-5

Villalba, C. (2003). El concepto de resiliencia individual y familiar. Aplicaciones en la intervención social. Psychosocial Intervention, 12(3), 283-299.

White, W.L. (2007). Selected Papers of William L. White. Journal of Addictive Diseases, 19(2), 1-10. Recuperado de: www.williamwhitepapers.com 
Anexo 1. Instrumento

\begin{tabular}{|c|c|c|c|c|c|c|}
\hline \multicolumn{7}{|c|}{ ISP.TS } \\
\hline & & $\begin{array}{l}\text { Casi siempre } \\
\quad 3\end{array}$ & & & & \\
\hline \multirow{6}{*}{ 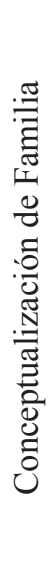 } & 1 & $\begin{array}{l}\text { Tomo en cuenta las experiencias histórico-sociales de los agentes en } \\
\text { cuestión, para entender como construyen su propia conceptualización de } \\
\text { familia }\end{array}$ & 1 & 2 & 3 & 4 \\
\hline & 2 & $\begin{array}{l}\text { Utilizo conceptualizaciones de familia desde otras disciplinas para arti- } \\
\text { cular con las que tienen las propias familias entrevistas }\end{array}$ & 1 & 2 & 3 & 4 \\
\hline & 3 & $\begin{array}{l}\text { Incorporo la concepción de familia de los agentes en cuestión para la } \\
\text { construcción de la Pericia Social. }\end{array}$ & 1 & 2 & 3 & 4 \\
\hline & 4 & $\begin{array}{l}\text { Utilizo conceptualizaciones de familia desde el Trabajo Social para arti- } \\
\text { cular con las que tienen las propias familias entrevistas }\end{array}$ & 1 & 2 & 3 & 4 \\
\hline & 5 & $\begin{array}{l}\text { Identifico las fortalezas, potencialidades y debilidades que la familia ex- } \\
\text { pone dentro de su propio funcionamiento. }\end{array}$ & 1 & 2 & 3 & 4 \\
\hline & 6 & $\begin{array}{l}\text { Utilizo conceptualizaciones de familia desde el ámbito legal articulando } \\
\text { con las de los propios agentes en cuestión. }\end{array}$ & 1 & 2 & 3 & 4 \\
\hline \multirow{6}{*}{ 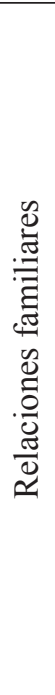 } & 7 & $\begin{array}{l}\text { Me centro en los roles de los miembros de la familia, generando énfa- } \\
\text { sis en los vínculos afectivos que las personas mantienen en su contexto } \\
\text { familiar. }\end{array}$ & 1 & 2 & 3 & 4 \\
\hline & 8 & $\begin{array}{l}\text { Observo los ejercicios de parentalidad, toma de decisiones, y vínculos } \\
\text { que se producen en la familia en los casos que corresponda. }\end{array}$ & 1 & 2 & 3 & 4 \\
\hline & 9 & $\begin{array}{l}\text { Observo las relaciones afectivas y sociales que se mantienen dentro del } \\
\text { sistema familiar de cohabitación }\end{array}$ & 1 & 2 & 3 & 4 \\
\hline & 10 & $\begin{array}{l}\text { Analizo como emergen nuevas dinámicas familiares y áreas de conflic- } \\
\text { tos potenciales, estudiando las relaciones entre sus miembros en vincu- } \\
\text { lación con su medio externo }\end{array}$ & 1 & 2 & 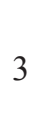 & 4 \\
\hline & 11 & $\begin{array}{l}\text { Focalizo en la relevancia de los patrones de interacción que los miem- } \\
\text { bros de la familia establecen, observando los sistemas de valores, creen- } \\
\text { cias y costumbres. }\end{array}$ & 1 & 2 & 3 & 4 \\
\hline & 12 & $\begin{array}{l}\text { Valoro la comunicación, el apoyo y el afecto mutuo, además de las si- } \\
\text { tuaciones de hostilidad o conflicto dentro del sistema familiar del agente } \\
\text { en cuestión }\end{array}$ & 1 & 2 & 3 & 4 \\
\hline \multirow{4}{*}{ 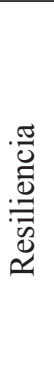 } & 13 & $\begin{array}{l}\text { Me centro en recopilar información y transformarla en fundamentos que } \\
\text { potencien las capacidades de los agentes en cuestión, aspecto que desta- } \\
\text { co en la apreciación profesional. }\end{array}$ & 1 & 2 & 3 & 4 \\
\hline & 14 & $\begin{array}{l}\text { Visualizo la resiliencia de los agentes en cuestión como un proceso de } \\
\text { superación de la adversidad y búsqueda de la autorrealización personal. }\end{array}$ & 1 & 2 & . & 4 \\
\hline & 15 & $\begin{array}{l}\text { Oriento mi observación en las fuentes de fortaleza, apoyo y resiliencia } \\
\text { familiar de los agentes en cuestión. }\end{array}$ & 1 & 2 & 3 & 4 \\
\hline & 16 & $\begin{array}{l}\text { Organizo la información del agente en cuestión desde el reconocimiento } \\
\text { del potencial de cada persona para alcanzar sus metas }\end{array}$ & 1 & 2 & 3 & 4 \\
\hline
\end{tabular}




\begin{tabular}{|c|c|c|c|c|c|c|}
\hline \multirow{6}{*}{ 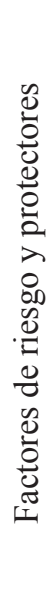 } & 17 & $\begin{array}{l}\text { Considero relevantes los vínculos al interior de la familia, estilos de } \\
\text { comunicación, estilos de resolución de conflictos y vínculos con redes } \\
\text { externas. }\end{array}$ & 1 & 2 & 3 & 4 \\
\hline & 18 & $\begin{array}{l}\text { Realizo proposiciones atendiendo a los factores de riesgos estáticos } \\
\text { identificados. }\end{array}$ & 1 & 2 & 3 & 4 \\
\hline & 19 & $\begin{array}{l}\text { Me centro en descubrir redes de apoyo que permitan intervenir en los } \\
\text { factores de riesgo del infractor de ley. }\end{array}$ & 1 & 2 & 3 & 4 \\
\hline & 20 & $\begin{array}{l}\text { En la recopilación de antecedentes individuales, familiares y penales, } \\
\text { relevando el fortalecimiento de habilidades personales y sociales del in- } \\
\text { fractor de ley. }\end{array}$ & 1 & 2 & 3 & 4 \\
\hline & 21 & $\begin{array}{l}\text { Realizo proposiciones de acuerdo con los factores de riesgos dinámicos } \\
\text { identificados. }\end{array}$ & 1 & 2 & 3 & 4 \\
\hline & 22 & $\begin{array}{l}\text { Visualizo la importancia de reconocer los factores protectores con la fi- } \\
\text { nalidad de que estos potencien las capacidades del agente en cuestión }\end{array}$ & 1 & 2 & 3 & 4 \\
\hline \multirow{6}{*}{ 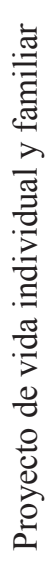 } & 23 & $\begin{array}{l}\text { Considero las posibilidades, factibilidades y oportunidades para la cons- } \\
\text { trucción de proyecto de vida del agente en cuestión. }\end{array}$ & 1 & 2 & 3 & 4 \\
\hline & 24 & $\begin{array}{l}\text { Considero que, a través de la obtención de una pena sustitutiva, se po- } \\
\text { sibilita desarrollar el proyecto de vida de una persona infractora de ley }\end{array}$ & 1 & 2 & 3 & 4 \\
\hline & 25 & $\begin{array}{l}\text { Considero que como perito Trabajador Social puedo contribuir al res- } \\
\text { tablecimiento de las redes necesarias para los proyectos de vida de las } \\
\text { personas condenadas a Penas Sustitutivas }\end{array}$ & 1 & 2 & 3 & 4 \\
\hline & 26 & $\begin{array}{l}\text { Considero la historia de vida y expresión de lo que desea ser a futuro el } \\
\text { agente en cuestión al momento de construir la Pericia Social }\end{array}$ & 1 & 2 & 3 & 4 \\
\hline & 27 & $\begin{array}{l}\text { En las Pericias Sociales valoro los proyectos de vida de los agentes en } \\
\text { cuestión en base a su propio contexto sociocultural }\end{array}$ & 1 & 2 & 3 & 4 \\
\hline & 28 & $\begin{array}{l}\text { Vislumbro las áreas de autoexpresión y autodesarrollo, relaciones inter- } \\
\text { personales, relaciones sociales y vida profesional del agente en cuestión. }\end{array}$ & 1 & 2 & 3 & 4 \\
\hline \multirow{4}{*}{ 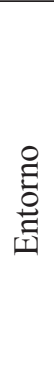 } & 29 & $\begin{array}{l}\text { Considero los riesgos del entorno y del vecindario como circunstancias } \\
\text { de vida donde se encuentra inmersa la familia del agente en cuestión. }\end{array}$ & 1 & 2 & 3 & 4 \\
\hline & 30 & $\begin{array}{l}\text { Reflexiono sobre la importancia de la estabilidad de la vivienda del agen- } \\
\text { te en cuestión como factor protector }\end{array}$ & 1 & 2 & 3 & 4 \\
\hline & 31 & $\begin{array}{l}\text { Observo las relaciones sociales de la familia del agente en cuestión en } \\
\text { su interacción con otras personas y en diferentes espacios del mismo } \\
\text { entorno }\end{array}$ & 1 & 2 & 3 & 4 \\
\hline & 32 & $\begin{array}{l}\text { Valoro que la conexión con su entorno permite mayor incidencia en } \\
\text { asuntos cívicos, culturales y de toma de decisiones }\end{array}$ & 1 & 2 & 3 & 4 \\
\hline
\end{tabular}




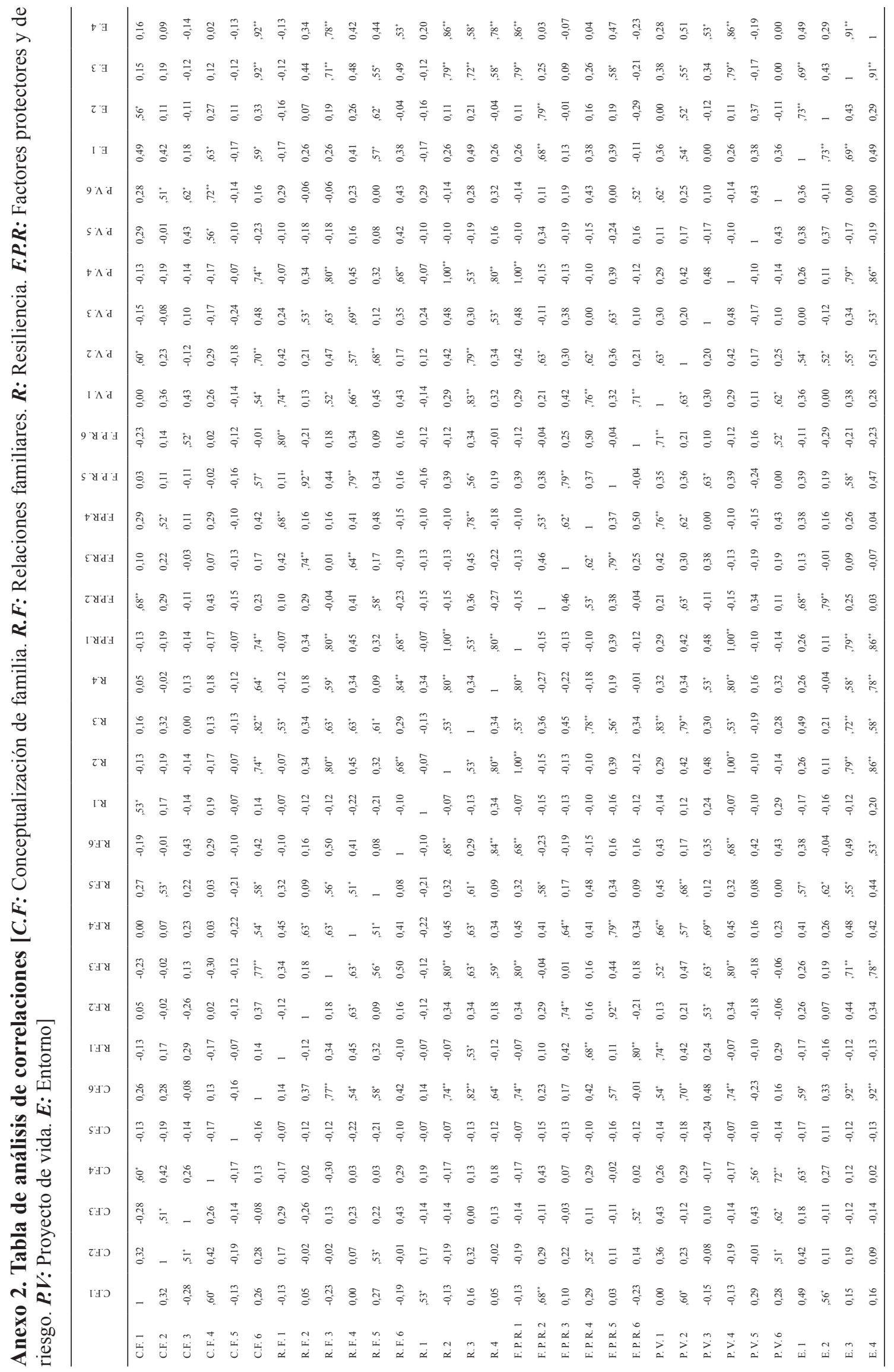

